\title{
Longterm appraisal of the histological appearances of the ileal reservoir mucosa after restorative proctocolectomy for ulcerative colitis
}

\author{
P Setti Carraro, I C Talbot, R J Nicholls
}

\begin{abstract}
Between November 1976 and December 1985,110 patients had restorative proctocolectomy for ulcerative colitis. The histological appearances in the reservoir mucosa were followed up in 60 of 109 survivors over 19-173 months (median 97). The median number of biopsy specimens taken per patient was six with a range of 3-13. These were examined by one pathologist (ICT) unaware of the clinical details using a scoring system previously described to assess the degree of chronic and acute inflammation. There was a significant correlation between the degree of severity of chronic and acute changes $(r=0.6192, p<0.000001)$. There was no correlation between the severity of inflammation and the following variables: preoperative duration of disease, presence of cancer or dysplasia in the original operative specimen, extra-alimentary manifestations or the type of reservoir. A significant correlation between severe inflammation and male sex was found $(p<0.035)$. The 60 patients could be divided into three groups based on the severity and fluctuation of histological inflammation. In group $A(n=27,45 \%)$ chronic changes were minor and acute inflammation was never seen. In group B $(n=25,42 \%)$ chronic changes were more severe and there were transient episodes of acute inflammation. In group $C(n=8$, $13 \%$ ) severe chronic and severe acute inflammation were constantly present. Differentiation of the three groups had clearly occurred within six months from closure of the ileostomy. Patients in group $C$ could be identified on histological criteria within weeks of closure of the ileostomy and were those exclusively at risk of developing chronic pouchitis. Chronic pouchitis never occurred in patients of groups $A$ and $B$. No case of dysplasia was seen. Histological assessment of the reservoir mucosa within a few months after closure of the ileostomy seems to define patients who will and who will not subsequently develop pouchitis. (Gut 1994; 35: 1721-1727)
\end{abstract}

London

P Setti Carraro
I C Talbot

R J Nicholls

Correspondence to: Mr R J Nicholls, St Mark's Hospital, City Road, London EC1V 2PS.

Accepted for publication 31 January 1994
Morphological changes in the mucosa of ileal reservoirs were first described in the Kock continent ileostomy. ${ }^{12}$ Philipson et al showed architectural changes compatible with increased cell turnover within a few weeks of construction of the reservoir. ${ }^{1}$ These were not associated with apparent functional impairment of the mucosa, ${ }^{1}$ ultrastructural changes ${ }^{1}$ or clinical dysfunction. ${ }^{3}$ They were considered to represent an adaptation of the ileal mucosa to the new environment. ${ }^{245}$ When followed up over periods ranging from two to 20 years a trend toward normality was reported. ${ }^{2} 67$ In no case was mucosal dysplasia noticed and severe acute inflammation of the reservoir was not seen by most investigators. ${ }^{1267}$

Since the introduction of restorative proctocolectomy, ${ }^{8}$ there has been a number of studies of the microscopic appearance of the mucosa of the ileal reservoir. ${ }^{49}{ }^{10-15}$ At a mean follow up of less than two years, mucosal changes similar to those seen in continent Kock ileostomies have been reported, ${ }^{512-15}$ affecting equally patients who originally had ulcerative colitis or familial adenomatous polyposis. ${ }^{12}$ In addition severe mucosal atrophy (colonic metaplasia) was reported in half of the biopsy specimens examined.591213 So far there has been no study reporting the fluctuations of the histological appearance of ileal reservoir mucosa over a period of years.

Kock first reported severe acute inflammation in a continent ileostomy. ${ }^{16}$ This, associated with clinical features of increased stool output, was subsequently called pouchitis. ${ }^{17}$ It was noticed 91112141819 that pouchitis spared patients originally affected by familial adenomatous polyposis, raising the possibility that a non-environmental factor within the reservoir might be responsible. Two clear cut types of mucosal adaptation occurring early after the closure of the ileostomy have been described. The first has shown stable features of mild chronic inflammation, the other those of progressive severe atrophy with an increasing degree of acute inflammation. ${ }^{10}$ In the second type the coexistence of colonic metaplasia and hyperproliferation might be expected to increase the neoplastic potential of the reservoir epithelium. ${ }^{20}$ To date, however, only one case of dysplastic change in an ileal reservoir mucosa has been reported. ${ }^{21}$

The first aim of this study was to see whether, in patients who had restorative proctocolectomy for ulcerative colitis, fluctuations occur in the morphological appearance of the reservoir mucosa. The second aim was to see whether there might be early morphological changes predicting the subsequent development of acute inflammation and therefore pouchitis. The third was to see whether dysplasia might occur with time. 
Patients and methods

Between January 1976 and December 1985, 110 patients had restorative proctocolectomy or proctectomy for ulcerative colitis at one hospital. There was one postoperative death $(0.9 \%)$. Of the 109 survivors, 60 had been followed up for a median period of 97 months (range 19-173). At follow up patients were questioned about function, including frequency of defecation, continence, the need for catheter evacuation, and any drugs taken. A mucosal biopsy specimen from the
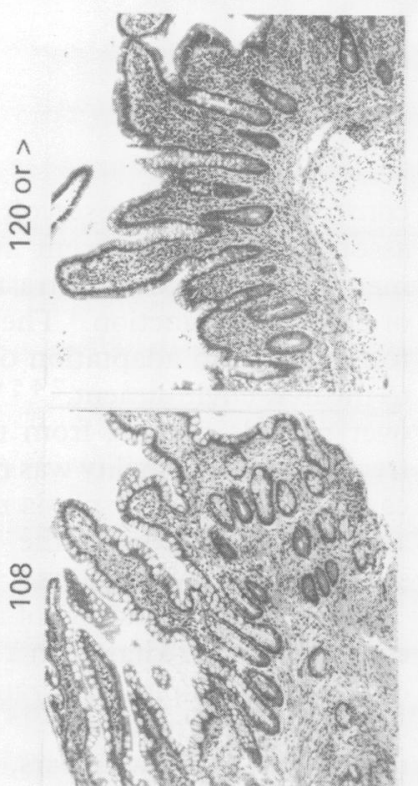

๑

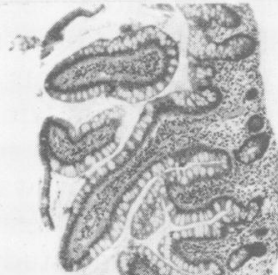

$\widehat{s}$
है
ह
है
है
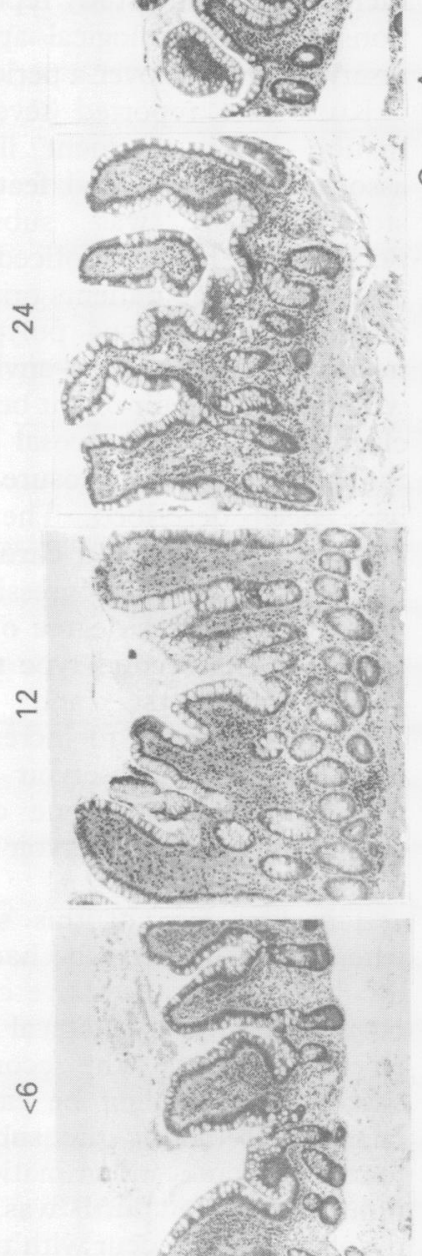
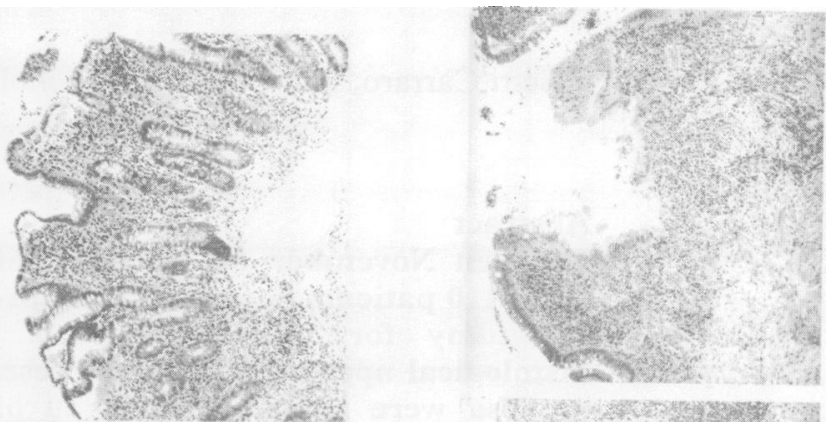


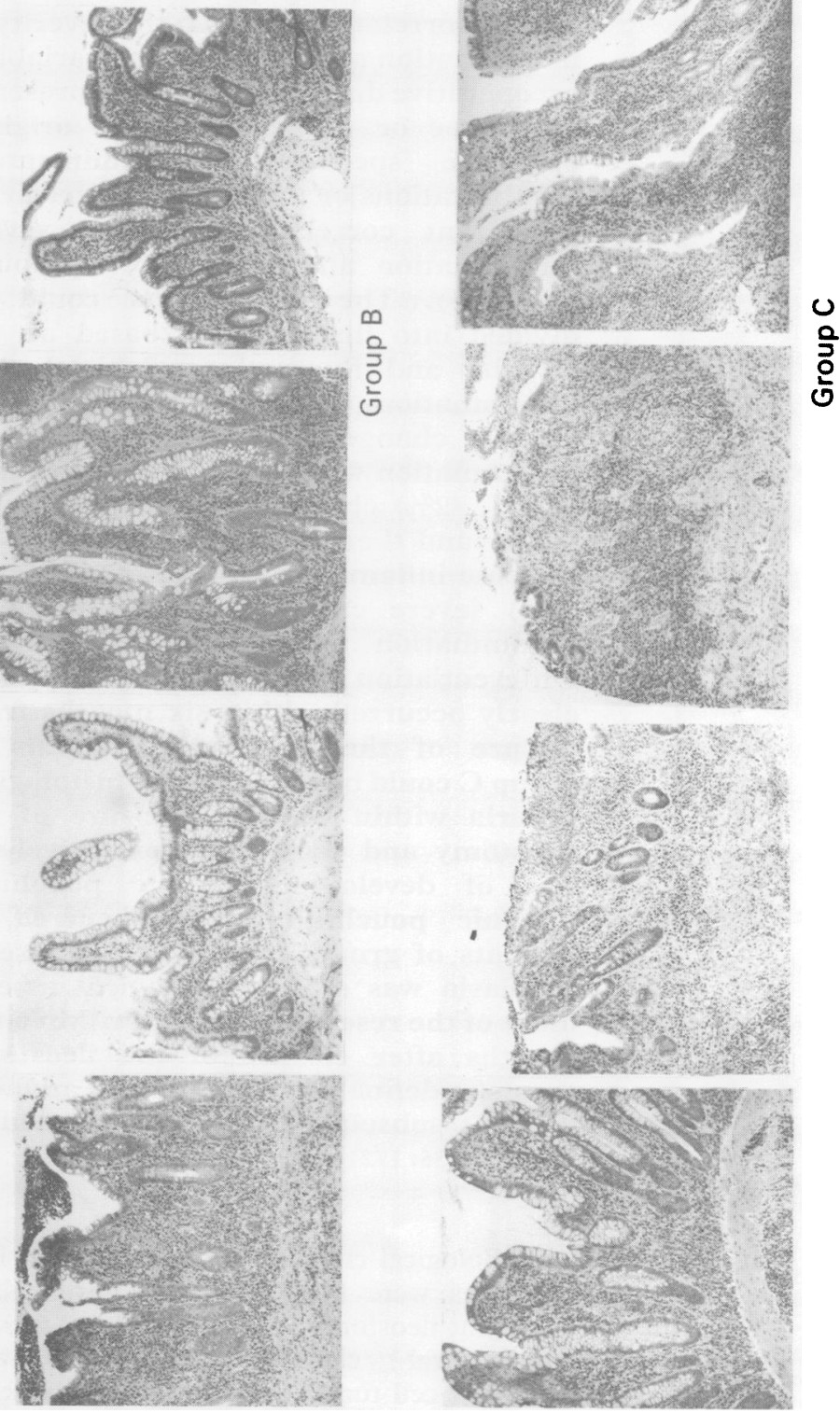


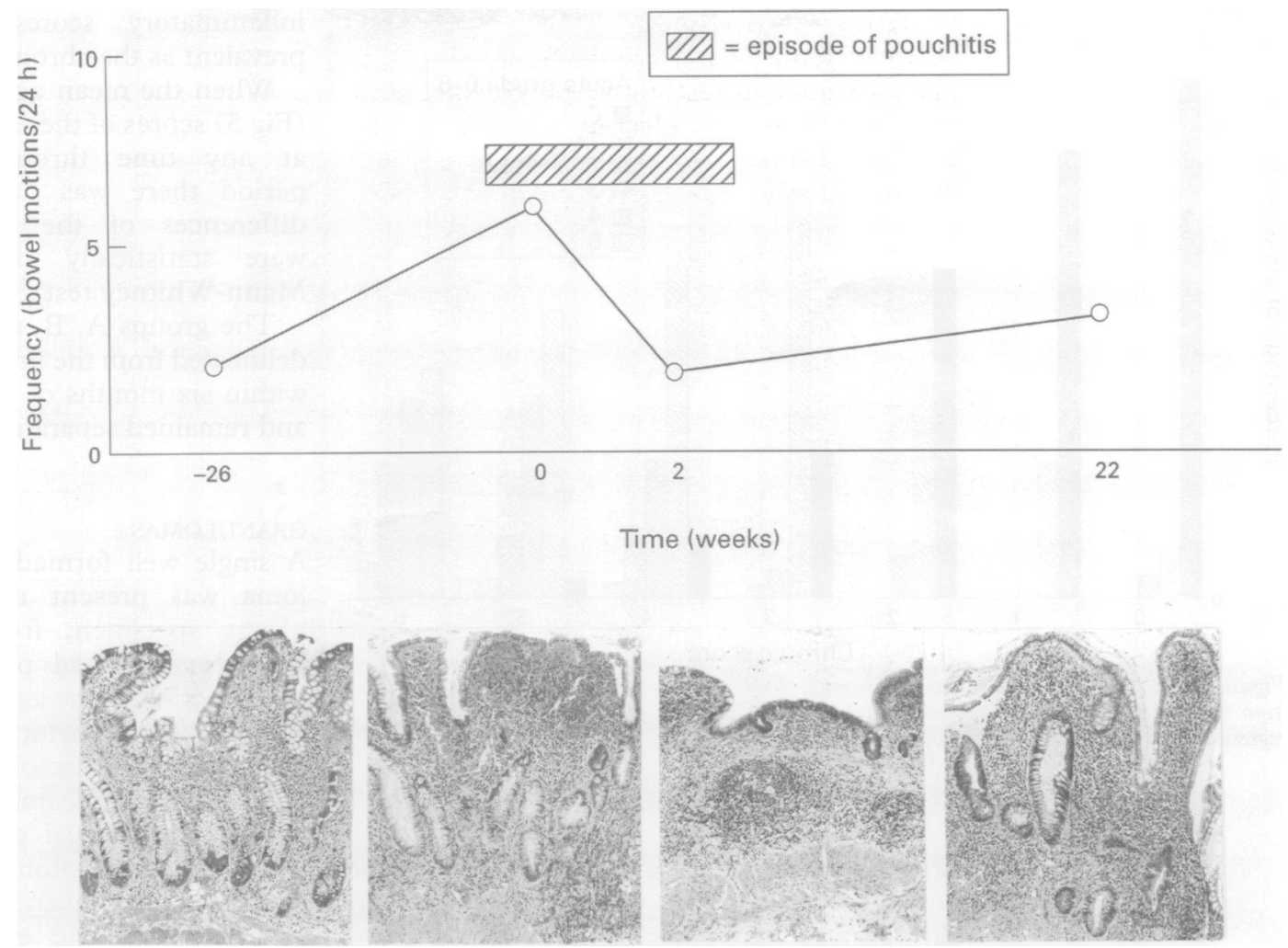

Figure 2: Typical changes in the reservoir mucosa during and after an episode of pouchitis in a patient from group B. After acute inflammatory changes there is recovery toward normality.

reservoir was taken during rigid sigmoidoscopy. There was thus a series of ileal mucosal biopsy specimens from the reservoir of each patient taken over a long period of time. A minimum of three reservoir mucosal biopsy specimens per patient was available (range 3-13 specimens per patient) with a median of six.

The biopsy specimen was orientated mucosa uppermost on a piece of ground glass or millepore and fixed in buffered formalin (10\%) for 24 hours. It was then embedded in paraffin wax. Sections were cut to a thickness of $4 \mu \mathrm{m}$ in the plane of the crypt glandular axis and stained with haematoxylin and eosin. They were examined by one pathologist (ICT) unaware of the patient's clinical details. The presence of histological features of acute and chronic inflammation ${ }^{9}$ was recorded and a score was allotted to each feature. This provided a numerical grade of severity of both acute and chronic inflammation for each specimen, designed grade Ac 0, 1, 2, 3-6 and grade $\mathrm{Ch} 0,1,2,3-6$ respectively.

There were 36 men and 24 women, with a mean age at presentation of 33 years (median 31 , range 14-60) and a mean age at onset of disease of 24 years (median 22, range 5 to 48 ). The mean duration of symptoms before restorative proctocolectomy was 8.8 years (median 6, range 0-31).

A three loop (S) reservoir was constructed in $25(42 \%)$, a two loop $(J)$ in $12(20 \%)$, and a four loop (W) in 23 (38\%). Fifty seven (95\%) patients had total or extensive and three left sided colitis. Dysplasia, either of low or high grade, ${ }^{22}$ was present in $14(23 \%)$ original operative specimens. This was associated with cancer in four cases. Information about postoperative function was retrieved from the follow up records.

A clinical diagnosis of pouchitis was made when patients complained of an increased frequency of defecation and the passage of watery stool combined with acute inflammation seen on rigid sigmoidoscopy and histological examination of a biopsy specimen (grade Ac 3). ${ }^{9}$

\section{STATISTICAL ANALYSIS}

The $\chi^{2}$ test was used to compare proportions for large samples and Fisher's exact test for small samples. Normally distributed data were compared by Student's $t$ test. Variables not normally distributed were compared using Wilcoxon's signed rank or Mann-Whitney tests. The relation between scores was tested using Spearman's rank correlation test and linear regression. Unless stated otherwise differences were regarded as significant for a probability value of less than $0 \cdot 05$.

\section{Results}

Three groups of patients could be distinguished. In the first, group A, minor mucosal histopathological changes of chronic and acute type (overall score acute + chronic 0 to 3 ) were present in all specimens throughout the follow up period with no evidence of a more severe grade at any time. There were $27(45 \%)$ patients in this group (Fig 1).

In $25(42 \%)$ patients, group $B$, there were features of more severe acute inflammation (grade Ac 2 to 5) at least once during the follow up period. These were always accompanied by severe chronic changes (grade $\mathrm{Ch} 4$ 


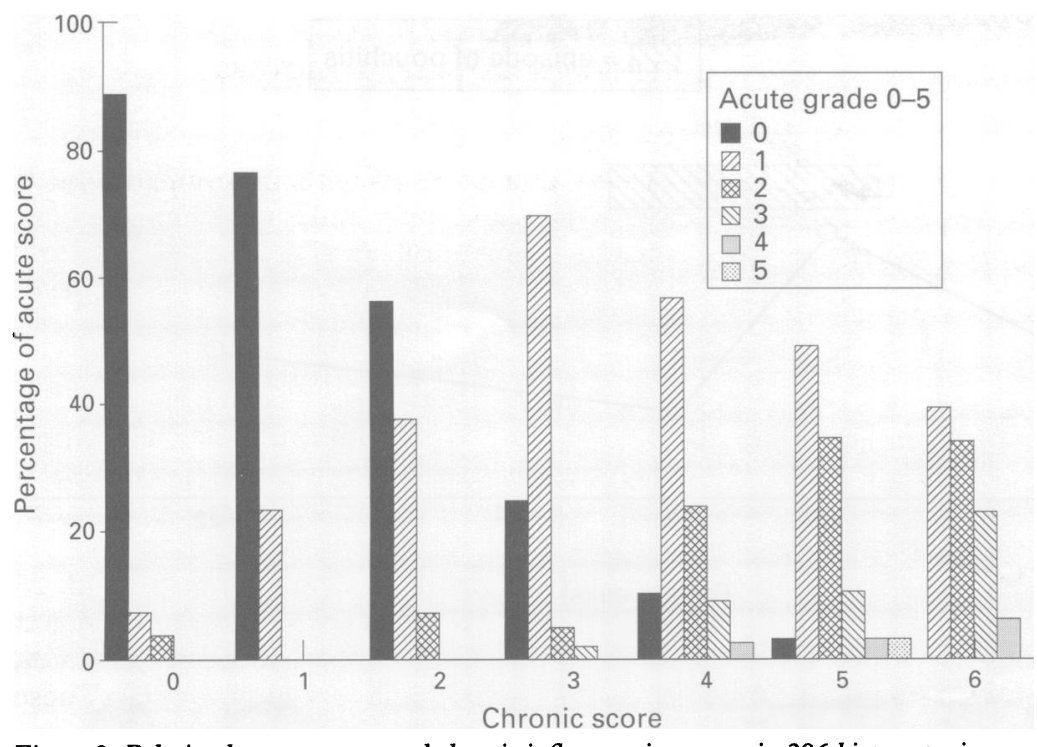

Figure 3: Relation between acute and chronic inflammation scores in 396 biopsy specimens from 60 patients. Spearman's correlation coefficient $=0.8728, p<0.00001$, two tailed; regression correlation coefficient $=0.6192, p<0.000001$.

to 6). Sequential biopsy specimens from the pouch in this group of patients, however, showed a return to normality or minor histological changes accompanying the recovery from an acute attack. Acute changes, namely neutrophil infiltrate, crypt abscesses, and mucosal ulceration, were always the first features to disappear while chronic severe changes could last for several months, usually in the form of partial villous atrophy (grade Ch 2) (Fig 1). During the follow up 13 patients showed these acute changes once and 12 at least twice (Fig 2).

Biopsy specimens from patients in groups A and B occasionally showed normal mucosa. Previous or subsequent specimens from the reservoir of these patients, however, always showed some degree of histopathological abnormality.

In the remaining eight $(13 \%)$ patients (group C) severe acute (grade Ac 2 to 5) and chronic (grade Ch 4 to 6 ) inflammation persisted without any recovery toward normality (Fig 1).

A highly significant correlation between the severity of acute and chronic inflammation was found (Fig 3). It can be seen that high acute

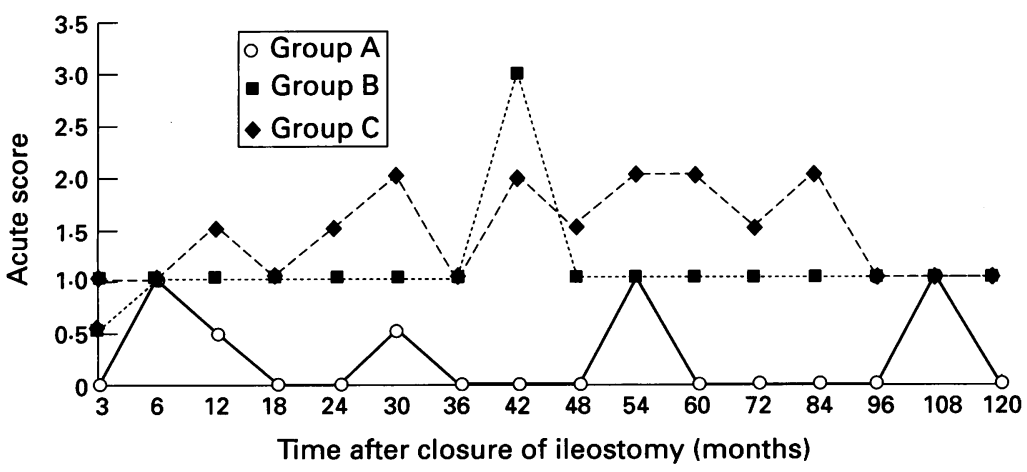

Figure 4: The grade of acute inflammation in the ileal reservoir mucosa after closure of the ileostomy in 60 patients. Values given are medians. $A$ v $B$ v $C, p<0.00001$, Mann-Whitney U test. inflammatory scores became increasingly prevalent as the chronic score rose.

When the mean acute (Fig 4) and chronic (Fig 5) scores of these groups were compared, at any time throughout the follow up period there was never any overlap. The differences of the medians at any time were statistically significant $(p<0.00001$, Mann-Whitney test).

The groups A, B, and C were each clearly delineated from the first biopsy specimen taken within six months of closure of the ileostomy and remained separate thereafter.

\section{GRANULOMAS}

A single well formed epithelioid cell granuloma was present in one pouch mucosal biopsy specimen, from a male patient of 17, who had had proctectomy and pouch construction at the age of 14 . There had been several years' history of ulcerative colitis, necessitating colectomy at the age of 10 . Histological appearance of the colon and rectum was typical of ulcerative colitis and there was no question of Crohn's disease. It is of interest, however, that there was a stitch granuloma in the excised rectum, related to the previous surgery and the patient eventually had to have his pouch excised, at the age of 18 , for refractory pouchitis. The excised pouch showed severe ulcerative pouchitis. There were syncyticial giant cells related to epithelial damage, but there were no granulomas or other features of Crohn's disease. No other granulomas were seen in this series, although we have encountered occasional isolated granulomas in pouch specimens from subsequent patients, without pouchitis or clinical problems.

FIBROSIS

Eighty six (22\%) biopsy specimens from 32 $(53 \%)$ patients showed fibrosis (Fig 6A). This was mild in 72 and dense in 14. It was seen in the lamina propria in 72 specimens and in the submucosa in 14. The true incidence of submucosal fibrosis could not be accurately assessed, however, because the submucosa was not always present in the biopsy specimens. When present, submucosal fibrosis was associated with mucosal (lamina propria) fibrosis in eight of 14 specimens.

Fibrosis was seen both early and late during the period of study. It was usually associated with a neutrophil infiltrate (grade Ac 1-2), moderate chronic cell infiltrate (grade $\mathrm{Ch} 2$ ), and subtotal villous atrophy (grade $\mathrm{Ch} 2$ ). It could be seen in sequential specimens from the same reservoir, and was not progressive. Fibrosis was not found to be related to the type of pouch (S 17 of 25, J 7 of 12, W 8 of 23, $p>0.05$ ), to sex (males 14 of 28 , females 10 of $32, p>0 \cdot 2$ ), to the occurrence of extraintestinal manifestations of inflammatory bowel disease $(p>0 \cdot 1)$ or to the presence of dysplasia and cancer in the original operative specimen $(p>0 \cdot 1)$. It correlated significantly with the occurrence of acute mucosal inflammation, 


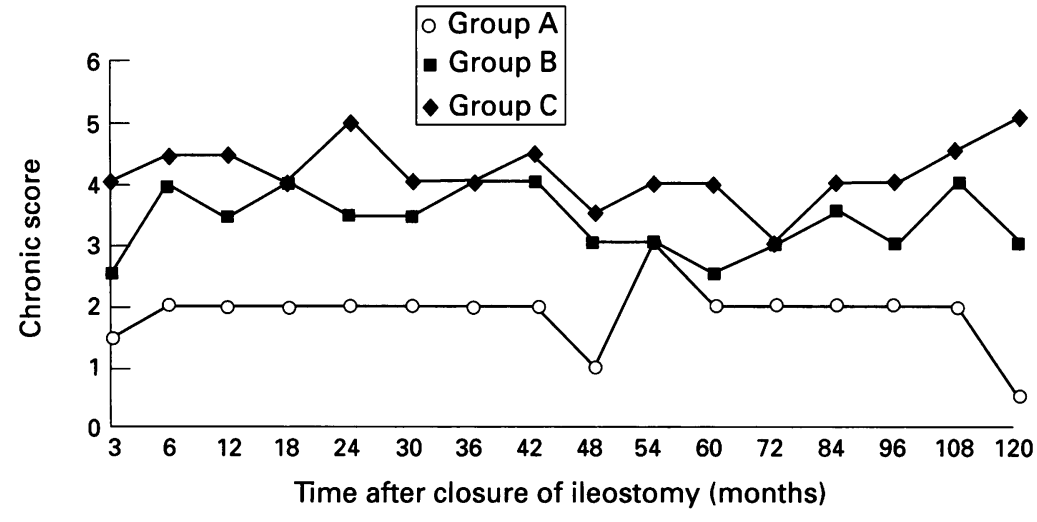

Figure 5: The grade of chronic inflammation in the ileal reservoir mucosa after closure of the ileostomy in 60 patients. Values given are medians. $A$ v $B$ v $C, p<0.00001$, Mann-Whitney U test.

\section{HISTOLOGY RELATED TO \\ CLINICOPATHOLOGICAL FACTORS}

Sex ratio

Twenty four of 33 patients in groups B and C were male and only nine were female. The corresponding numbers of group A were 12 and 15 respectively $(p=0 \cdot 035$, Fisher's exact test).

\section{Preoperative factors}

There was no significant difference in the preoperative duration of symptoms in groups B and $C$ compared with those in group $A$ (mean 9.8 years $v 7.5$ years respectively, $\mathrm{p}>0.2$ ).

Backwash ileitis was present in four of 18 operative specimens in which terminal ileum was present. Two of these were in group A, none in group B, and two in group C. These differences were not significant $(p=0 \cdot 7)$.

There was no significant correlation between the presence of cancer in the operative specimen nor of extraintestinal manifestations of inflammatory bowel disease before or after surgery and the groups $\mathrm{A}, \mathrm{B}$, or C.

Dysplasia was present in the operative specimen of six $(24 \%)$ of 25 patients in group B and in four $(50 \%)$ of eight of group C, but only in four (15\%) of 27 of group A. While suggesting a possible trend, this difference was not statistically significant $(p=0 \cdot 11)$.

\section{DYSPLASIA}

No case of dysplasia was seen in any of the biopsy specimens.
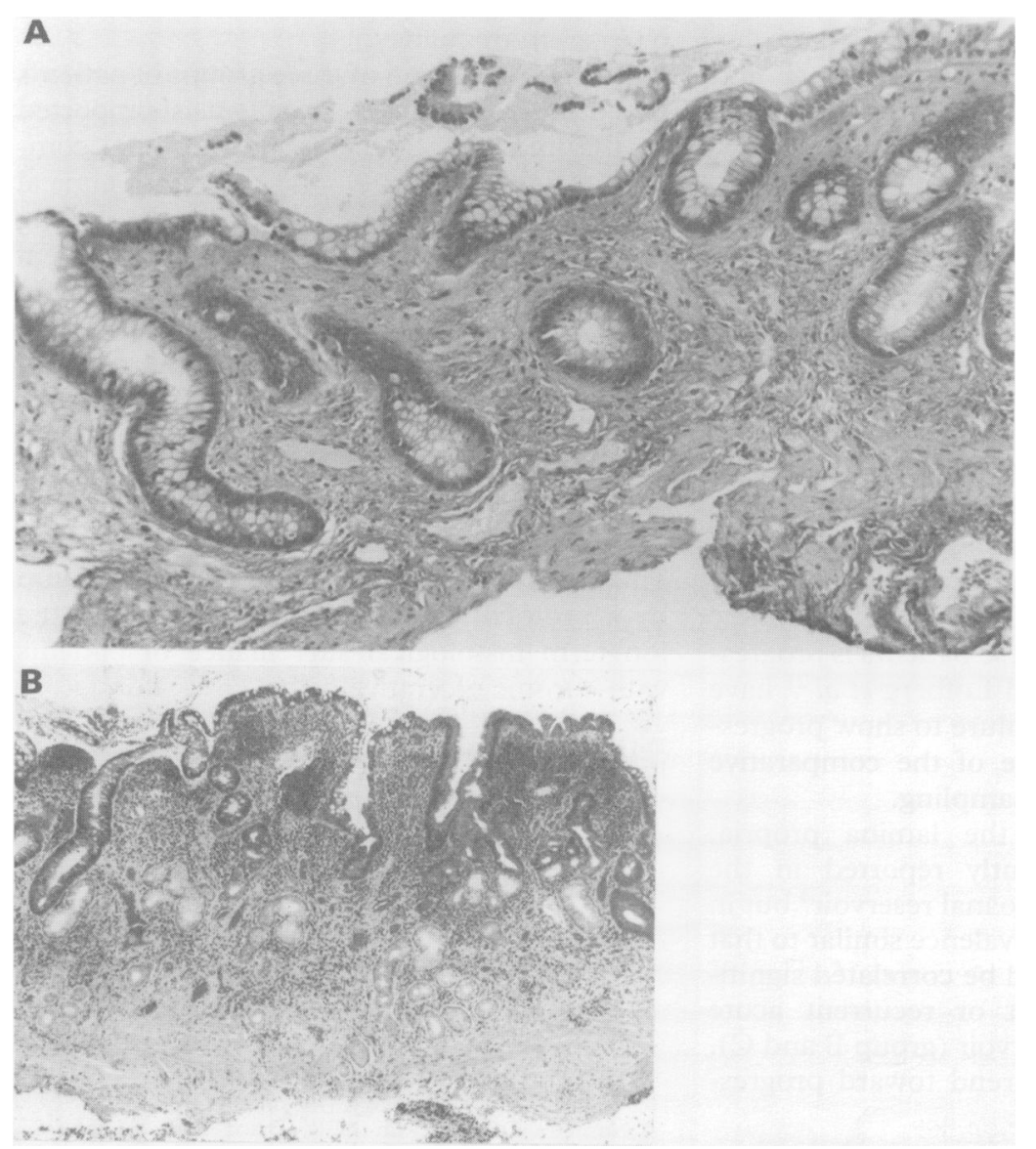

Figure 6: (A) Typical appearance of mucosal and submucosal fibrosis of the ileal reservoir; (B) pseudopyloric metaplasia was occasionally seen during acute inflammation of the pouch.

\section{Postoperative factors}

Patients in group A never developed pouchitis. Conversely pouchitis occurred in 24 of 33 in groups $\mathrm{B}$ and $\mathrm{C}$. In nine patients in group $\mathrm{B}$ microscopic acute inflammation of the reservoir occurred without changes in frequency of defecation, but all patients in group $C$ were symptomatic and suffered from chronic unremitting pouchitis.

Patients in group A had a mean number of bowel movements in 24 hours of $3 \cdot 8$ (range $1-6)$. The respective rates in group $B$ and $C$ were 5.9 (range 2-11) and 4.3 (range 1-12). The difference was significant for group A $v$ B $(\mathrm{p}=0.001)$, but not for $\mathrm{A} v \mathrm{C}$ and $\mathrm{C} v \mathrm{~B}$. There was no significant difference between pouch type and histological group.

\section{Discussion}

The experience with the Kock ileostomy has shown that most patients with ulcerative colitis have abnormal mucosa in the reservoir. ${ }^{1-367}$ In this study there has been no case in which seen. Even in the small proportion of those having a normal biopsy specimen on one occasion, others, in that patient, have been abnormal. The results overall mirror those reported for the Kock ileostomy.

This study has, however, yielded some new information. We found that there are varying degrees of chronic and acute changes in the patients. Based on the severity of inflammation it has been possible to place them into one of three groups (A, B, C). The groups are evident from within a few months $(<6)$ from closure of normal appearances have been constantly 
the ileostomy and have remained separate up to the final point of sampling at a minimum of five years. At no time have patients in groups A, B, or C overlapped. Furthermore, the groups can be correlated with clinical outcome. Episodes of pouchitis have never occurred in group A. Pouchitis has been present permanently in group $\mathrm{C}$ and has been seen in group B only in association with histological features of acute inflammation. In this group histological recovery is associated with clinical resolution.

Veress et al in a series of 77 patients came to a similar classification of histological appearances. ${ }^{23}$ Their mean follow up was, however, slightly less at 4.8 years. They differentiated patients with biopsy specimens showing villous atrophy, which in their system was called group B, but in ours seems to be equivalent to group $\mathrm{C}$. In both experiences the proportion of patients in either of these groups was about $10 \%$ of the population studied. These patients had increased inflammatory changes, acute as well as chronic, associated with villous atrophy. Of considerable interest, they found three of the total number of eight patients in their group B to have some form of dysplasia. Of these, two had changes indefinite for dysplasia and one low grade true dysplasia. In our eight patients in group $C$, none had dysplasia but if the group B of Veress and the group $\mathrm{C}$ of this study are equivalent, the inference is clear that these patients need regular, frequent follow up with biopsy examination. This study has shown that this possibly high risk group can be identified from the outset that is, early after closure of the ileostomy.

The correlation seen between acute and chronic changes is in line with previous reports. ${ }^{9} 18$ Patients probably have a variable reaction in the form of a differing acute inflammatory response to the changed environment provided by the contents of the reservoir. ${ }^{245}$ These acute changes might then trigger an enhanced hyperproliferative response of the ileal mucosa in the form of increased crypt cell turnover, ${ }^{1614}$ associated with villous atrophy. In this study the hyperproliferative response seems to last for months, while acute changes disappear quite rapidly. In some patients acute changes recur and the enhanced cell turnover within the crypts persists, leading to severe villous atrophy. Although we could not show that villous atrophy in these patients is progressive, Veress et al ${ }^{1023}$ and Lofberg et al ${ }^{21}$ have shown it to be so. Our failure to show progression is probably because of the comparative infrequency of mucosal sampling.

Fibrosis, usually of the lamina propria, has been more frequently reported in the Kock $^{1267}$ than in the ileoanal reservoir ${ }^{4}$ but in our series there was a prevalence similar to that seen in the first. ${ }^{7}$ It could be correlated significantly with intermittent or recurrent acute inflammation of the reservoir (group B and C). There was no obvious trend toward progression of fibrosis with time.

Pseudopyloric metaplasia is regarded to be a response to small intestinal mucosal inflammation. ${ }^{24}$ In this study it was always associated with acute inflammatory changes, occurring more frequently in patients with recurrent episodes of inflammation of the reservoir. This feature might be a useful sign pointing to previous active pouchitis.

The only clinical factor significantly associated with recurrent episodes of acute inflammation of the pouch was male sex. This finding has been made by others (personal communication). Whether there is an enhancing or protective hormonal factor of whether other sex dependent or associated factors play a part is a matter for further study.

A relation between dysplasia in the operative specimen and tendency to hyperproliferative responses in the reservoir might be expected. Patients with dysplasia in the operative specimen tended to fall into the $B$ or $C$ categories. The difference in prevalence in the different categories, however, failed to reach statistical significance, but clearly further study of this important question is necessary.

The results of this study only partially confirmed the finding of Moskowitz et al ${ }^{9}$ that the degree of histological inflammation could be correlated with frequency of defecation. This was true when group A was compared with groups $B$ and $C$ combined, it was not when group $\mathrm{A}$ was compared with group $\mathrm{C}$ alone. The number of patients in group $\mathrm{C}$ was small and all were receiving high doses of antidiarrhoeal drugs, which may explain the lack of significant correlation between these groups. Nine of 25 patients in group B, however, showed histological features of severe mucosal inflammation occurring without corresponding changes in frequency.

The identification of three groups of patients based on histological assessment is supported by the findings of others, ${ }^{1023}$ (personal communication). If true, then it has clinical relevance. Patients at risk of developing unremitting pouchitis (group C) would seem to be identifiable from within a short time from closure of the ileostomy. It is also possible that those at risk of developing dysplasia also fall within this group. While this study in the intermediate term (5-10 years from ileostomy closure) has shown no case of dysplasia, the results from Veress et al 102123 show that patients with persisting acute and chronic inflammation (group C) should be followed up by a protocol similar to that adopted for the longterm histological surveillance of patients with chronic ulcerative colitis. ${ }^{25}$

PSC was supported by Ospedale Maggiore Policlinico, Milan, Italy.

1 Philipson B, Brandberg A, Jagenburg R, Kock NG, Lager I, Ahren C. Mucosal morphology, bacteriology, and absorption in intra-abdominal ileostomy reservoir. Scand $\mathfrak{f}$ Gastroenterol 1975; 10: 145-53.

2 Nilsson LO, Kock NG, Lindgren I, Myrvold HE, Philipson BM, Ahren C. Morphological and histochemical changes in the mucosa of the continent ileostomy reservoir 6-10 years after its construction. Scand $\mathcal{F}$ Gastroenterol 1980; 15: $737-47$

3 Kelly DG, Phillips SF, Kelly KA, Weinstein WM, Gilchrist MJR. Dysfunction of the continent ileostomy: clinical features and bacteriology. Gut 1983; 24: 193-201.

4 Nasmyth DG, Godwin PGR, Dixon MF, Williams N, Johnston D. Ileal ecology after pouch-anal anastomosis or ileostomy. A study of mucosal morphology, fecal bacteriology, fecal volatile fatty acids, and their interrelationship. Gastroenterology 1989; 96: 817-24. 
5 Luukkonen P, Valtonen V, Sivonen A, Sipponen P, Jarvinen H. Fecal bacteriology and reservoir ileitis in patients operated on for ulcerative colitis. Dis Colon Rectum 1988; 31: 864-7.

6 Go PMNYH, Lens J, Bosman FT. Mucosal alterations in the reservoir of patients with Kock's continent ileostomy. Scand F Gastroenterol 1987; 22: 1076-80.

7 Ojerskog B, Kock NG, Nilsson LO, Philipson BM, Ahren C. Long term follow-up of patients with continent C. Long term follow-up of patients with

8 Parks AG, Nicholls RJ. Proctocolectomy without ileostomy for ulcerative colitis. BMF 1978; 2: 85-8.

9 Moskowitz RL, Shepherd NA, Nicholls RJ. An assessment of inflammation in the reservoir after restorative proctocolectomy with ileoanal ileal reservoir. Int $\mathcal{F}$ Colorectal Dis 1986; 1: 167-74.

10 Veress B, Reinholt FP, Lindquist K, Liljeqvist L. Different types of mucosal adaptation in the ileal reservoir after restorative proctocolectomy. APMIS 1990; 98: 786-96.

11 Nicholls RJ, Belliveau P, Neill $M$, Wilks $M$, Tabaqchali $S$. Restorative proctecolectomy with ileal reservoir: a pathophysiological assessment. Gut 1981; 22: 462-8.

12 Shepherd NA, Jass JR, Duval I, Moskowitz RL, Nicholls RJ, Morson BC. Restorative proctocolectomy with ileal reservoir: pathological and histochemical study of reservoir: pathological and histochemical study of
mucosal biopsy specimens. $\mathcal{F}$ Clin Pathol 1987; 40: 601-7.

13 O'Connell PR, Rankin DR, Weiland LH, Kelly KA Enteric bacteriology, absorption, morphology and emptying after ileal pouch-anal anastomosis. Br f Surg 1986; 73: 909-14.

14 de Silva HJ, Millard PR, Kettlewell M, Mortensen NJ, Prince C, Jewell DP. Mucosal characteristics of pelvic ilea pouches. Gut 1991; 32: 61-5.

15 Reinholt FP, Veress B, Lindquist K, Liljeqvist L.
Qualitative assessment and morphometry in the study of the ileal reservoir after restorative proctocolectomy. APMIS 1989; 97: 97-104.

16 Kock NG, Darle N, Nulten L, Keventer J, Myrvold H, Phillipson B. Ileostomy. Curr Prob General Surg 1977; 14: 36-8.

17 Anonymous. Conventional versus continent ileostomies [Editorial]. Lancet 1980; i: 292-3.

18 Nicholls RJ, Moskowitz RL, Shepherd NA. Restorative proctocolectomy with ileal reservoir. Br f Surg 1985; 72: proctoco.

19 Hulten L, Svaninger G. Facts about the Kock continent ileostomy. Dis Colon Rectum 1984; 27: 553-7.

20 Shepherd NA. The pelvic ileal reservoir: apocalypse later? BMf 1991; 301: 886-7.

21 Lofberg R, Lilieqvist L, Lindquist $\mathrm{K}$, Veress B, Reinholt FP, Tribukait B. Dysplasia and DNA aneuploidy in a pelvic pouch. Report of a case. Dis Colon Rectum 1991; 34: $280-4$.

22 Riddel RH, Goldman H, Ransohoff DF, et al. Dysplasia in inflammatory bowel disease: standardized classification 14: 931-68.

23 Veress B, Reinholt FP, Lindquist K, Liljeqvist L. Mucosal adaptation in the ileal reservoir after restorative proctocolectomy. A long term follow-up study. [Abstract.] Ann Chir 1992; 46: 8 .

24 Wright NA, Pike C, Elia G. Induction of a novel epidermal growth factor-secreting cell lineage by mucosal ulceration in gastrointestinal stem cells. Nature 1990; 343: 82-5.

25 Lennard-Jones JE, Melville DM, Morson BC, Ritchie JK, Williams CB. Precancer and cancer in extensive ulcerative colitis: findings among 401 patients over 22 years. Gut 1990 ; 31: 800-6. 